\title{
Stammzellforschung
}

\section{Makrophagen in der Krankheitsentstehung und Therapie}

\author{
NICO LACHMANN \\ KLINIK FÜR PÄDIATRISCHE PNEUMOLOGIE, ALLERGOLOGIE UND NEONATOLOGIE, \\ MEDIZINISCHE HOCHSCHULE HANNOVER
}

\section{Macrophages are unique cells of the innate immunity and can be found in various tissues (tissue resident macrophages; TRMs). Macrophages and TRMs play a pivotal role in tissue homeostasis and can contribute to the onset and progression of certain diseases. We and others have developed macrophage-based cellular immunotherapies and explored the field of immune cell farming using induced pluripotent stem cells (iPSCs). Combination of iPSCs and bioreactors opens new ways in cellular therapies.}

DOI: $10.1007 / \mathrm{s} 12268-021-1547-\mathrm{y}$

(C) Der Autor 2021

Makrophagen spielen eine wesentliche Rolle in der angeborenen Immunität und sind ein wesentlicher zellulärer Bestandteil bei der Homöostase unterschiedlicher Organe. So kommen Makrophagen als gewebsständige Zellen im Gehirn (Mikroglia), der Lunge (Alveolarmakrophagen), der Leber (KupfferZellen) und weiteren Organen vor. Im klassischen Sinne entstehen Makrophagen aus im peripheren Blut patrouillierenden Monozyten. Man hat lange Zeit angenommen, dass gewebsständige Makrophagen ebenfalls aus Monozyten hervorgehen, was jedoch nicht für alle Gewebsmakrophagen zutrifft. In einem gesunden Organismus werden bereits vor der Geburt die Makrophagen, z. B. im Gehirn oder auch in der Lunge, unabhängig von den Blutstammzellen angelegt. Aufgrund von Genexpressionsprofilen, die ähnlich zu embryonalen Stammzellen sind, haben diese Gewebsmakrophagen die Fähigkeiten, den eigenen Bestand größtenteils autonom zu erneuern. Je nach Organ bzw. Gewebe findet jedoch unter homöostatischen Bedingungen in unterschiedlichen Intervallen ein Austausch der Makrophagen durch Monozyten aus der Peripherie statt. Bei Störungen - wie etwa genetischen Defekten - kann es zu einer gestörten Entstehung und/oder Funktion der Makrophagen in einzelnen Organen kommen.
So führen Mutationen im Rezeptor für den Granulozyten-Makrophagen-Kolonie-stimulierenden Faktor (GM-CSFR) zu einer gestörten Entstehung von Alveolarmakrophagen, was zum Krankheitsbild der pulmonalen Alveolarproteinose (PAP) führt [1]. Alveolarmakrophagen sind ein wesentlicher zellulärer Bestandteil bei der Bekämpfung von unerwünschten Eindringlingen in der Lunge. Darüber hinaus balancieren Alveolarmakrophagen das Surfactant, indem sie überschüssiges Surfactant abbauen. Aufgrund der zentralen Rolle des Granulozyten-MakrophagenKolonie-stimulierenden Faktors (GM-CSF) für die Aktivierung von Alveolarmakrophagen und den Abbau des Surfactants führen Mutationen im GM-CSFR zu einer Störung dieses Prozesses. Als Konsequenz sammelt sich im bronchoalveolären Raum Surfactant an, welches letztendlich den Gasaustausch behindert. Dieses Krankheitsbild kommt in Deutschland äußerst selten vor (ca. 1 in 10.000.000). Eine kausale Therapie gibt es für die erblich bedingte Alveolarproteinose derzeit nicht, sodass bei PAP-Patienten, welche an dieser Form leiden, mittels einer Lungenspülung (bronchoalveoläre Lavage) das Surfactant beseitigt und die Lunge gereinigt wird [1]. Diese Prozedur muss teils monatlich durchgeführt werden und ist für den Patienten sehr belastend. Daher wollen wir gemein- sam mit weiteren Partnern direkt an der Ursache, den Alveolarmakrophagen, angreifen und daraus neue Therapien entwickeln.

Neben Mutationen, die zu einer gestörten Entwicklung von Alveolarmakrophagen führen, gibt es ebenso Mutationen, die direkt die Funktion der reifen Zellen beinträchtigen. Mutationen im Interferon-gamma-Rezeptor (IFNgR) führen z. B. zu funktional beeinträchtigten Makrophagen, welche sich resultierend im Krankheitsbild der mendelian susceptibility to mycobacterial disease (MSMD) und somit in einer Anfälligkeit für mykobakterielle Erreger darstellen [2]. Alveolarmakrophagen kommen als erster Zelltyp direkt mit unterschiedlichen Erregern, so auch Mykobakterien, in Kontakt. In enger Zusammenarbeit mit weiteren Immunzellen sind diese dann in der Lage, den Erreger zu bekämpfen. Ist dieses Zusammenspiel jedoch z. B. durch Mutation des IFNgR auf Makrophagen gestört, kann kein IFNg auf deren Oberfläche binden und der Abbau der Erreger nicht stattfinden. Klinisch äußert sich diese Erkrankung durch teils sehr schwer verlaufende Lungeninfektionen, die zumeist mit geeigneten Antibiotika therapiert werden müssen. Da es sich jedoch um einen genetischen Defekt handelt, sind diese Infektionen nur schwer therapierbar und in den häufigsten Fällen wiederkehrend, was zum Teil letal enden kann. Auch für Patienten, die an einer IFNgR-defizitären MSMD leiden, gibt es derzeit kaum kausale Therapieoptionen. Aufgrund der zentralen Bedeutung von Makrophagen bei MSMD haben wir auch bei dieser Erkrankung neue therapeutische Wege mit Makrophagen untersucht.

Wie bereits zuvor erwähnt, spielen Makrophagen auch in anderen Organen eine wesentliche Rolle, sodass unterschiedlichste genetische Veränderungen auch hier die Entstehung sowie Funktion von Makrophagen stark beeinflussen und zu Erkrankungen führen können.

\section{Makrophagen-basierte Therapien}

Aufgrund der zentralen Bedeutung von Makrophagen in der Krankheitsentstehung 


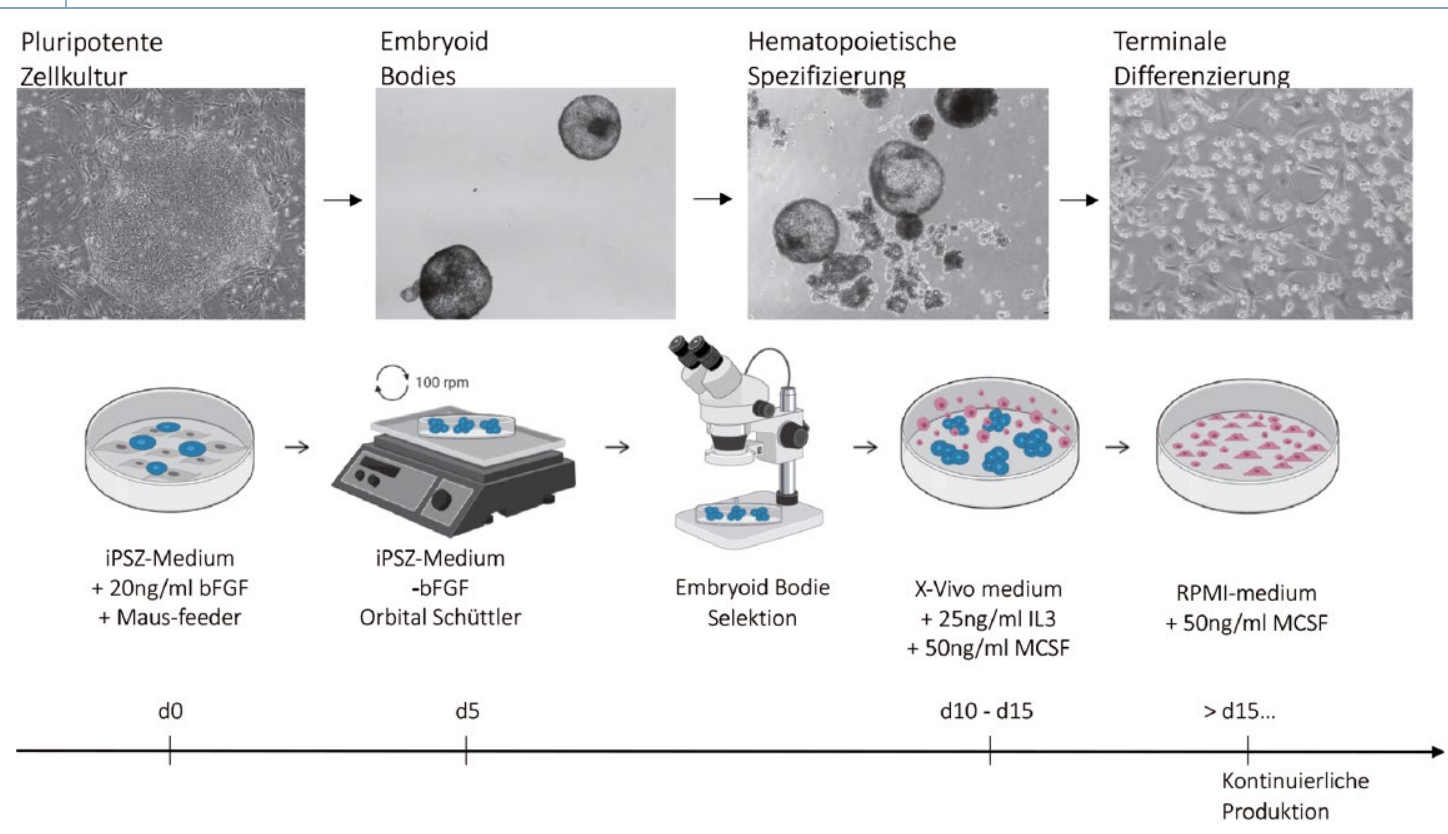

$\Delta$ Abb. 1: Schematischer Überblick zur Herstellung von Makrophagen aus humanen iPSZ. Die iPSZ werden zunächst auf murinen Feederzellen kultiviert, wonach die Zellen dann innerhalb von fünf Tagen zu embryoid bodies (EBs) auf einem Orbitalschüttler differenziert werden. Im Anschluss werden die EBs selektiert und im Spezifizierungsmedium für 10-15 Tage weiter differenziert. Ab dem Tag 15 können die Monozyten/Makrophagen kontinuierlich in einem wöchentlichen Turnus aus dem Überstand geerntet werden und weiter ausdifferenziert werden. Die Abbildung wurde zum Teil mit Biorender.com erstellt. von dysfunktionalen Makroph a ge n, haben wir SwaPI (swapping the pulmonary immunity) entwickelt, bei dem in einem einfachen Verfahren dysfunktionale Makrophagen direkt mit gesunden Makrophagen innerhalb der Lunge ausgetauscht werden. Die Grundlagen und weitere Optimierungen werden derzeit an Alveolarmakrophagen vorgenommen, sollen aber in naher Zukunft auf weitere Organsysteme übertragen werden. haben wir und andere neue und langanhaltende Makrophagen-basierte Therapien entwickelt. Wie bereits zuvor erwähnt, ist die Entstehung und Funktion von Alveolarmakrophagen bei der Alveolarproteinose gestört. Da die Zellen in der Lunge fehlen, hat sich der direkte Ersatz von Makrophagen in der Lunge durch eine intra-pulmonale Transplantation dieser Zellen als ein vielversprechender Ansatz bewährt. Ausgehend von hämatopoetischen Stammzellen (HSZ) haben wir diese im Mausmodell mittels gentherapeutischer Verfahren korrigiert und im Anschluss zu Makrophagen differenziert. Die so hergestellten Zellen wurden dann durch einen Tubus direkt in die Lunge von erkrankten Mäusen gegeben, ähnlich dem Vorgehen bei Patienten [3, 4]. Diese einfache Prozedur erweist sich als äußerst schnell sowie effizient. Nach der einmaligen Gabe der Zellen konnten wir beobachten, dass die verabreichten Makrophagen ausschließlich in der Lunge zu finden sind und diese dort mehr als zwölf Monate verweilen [3]. Die langanhaltende Persistenz der Zellen geht mit einer eindrucksvollen Verbesserung des PAPKrankheitsbilds einher. So konnten wir in unterschiedlichen radiologischen und histopathologischen Untersuchungen nachweisen, dass sich die Konzentration von Surfactant sowie weitere Parameter fast normalisieren. Auch eine normalisierte Lebenserwartung der Tiere ähnlich zu gesunden Vergleichstieren konnten wir beobachten [4].
Aufgrund dieser Daten haben wir damit begonnen, Genfähren zu entwickeln, die es uns erlauben, den genetischen Defekt des GM-CSF-Signalwegs in menschlichen Zellen zu korrigieren [5]. Da auch diese Versuche erfolgreich durchgeführt wurden, steht nun eine klinische Phase-I-Studie am Cincinnati Childrens Hospital Medical Center (Prof. Bruce Trapnell) an, um zunächst die Sicherheit von patienteneigenen gentechnischkorrigierten Makrophagen in PAP-Patienten zu untersuchen.

Um die therapeutischen Möglichkeiten von Makrophagen weiter zu untersuchen, haben wir ebenfalls genetisch-korrigierte HSZ in ein IFNgR-defizitäres Mausmodell der MSMD transplantiert [6]. Erste Untersuchungen konnten zeigen, dass die Mäuse nach einer Mycobacterium bovis-Bacillus-CalmetteGuérin(BCG)-vermittelten Immunisierung keinerlei Symptome aufzeigten und dass die Mäuse phänotypisch gleich zu gesunden Vergleichstieren waren. Dezidierte Untersuchungen und die Verwendung von Genfähren, die das korrigierte Gen nur in Makrophagen exprimieren, konnten aufzeigen, dass Alveolarmakrophagen in den Lungen von IFNgR-defizitären Tieren zu finden sind und dass diese Zellen der Grund für eine korrigierte und stabile Immunabwehr gegen BCG sind [6].

Diese erfolgreichen Studien haben wir nun in eine komplett neue Form der zellbasierten Immuntherapie übernommen. Ausgehend

\section{Neuste Entwicklung in der Herstellung von Makrophagen}

Als Alternative zu HSZ haben wir in den letzten Jahren neue Zelltechnologien, wie die induzierten pluripotenten Stammzellen (iPSZ), für die Generierung von Makrophagen verwendet. Der Vorteil: iPSZ lassen sich von jedem Spender generieren und können unbegrenzt in Kultur vermehrt werden. Ausgehend von gesunden iPSZ haben wir eine Plattform entwickelt, die es uns erlaubt, Makrophagen sowie andere Immunzellen in einem kontinuierlichen Herstellungsprozess zu erzeugen. Dabei werden die iPSZ in ca. fünf bis zehn Tagen zu einem Zellaggregat (embryoid bodie, EB) differenziert, welcher im weiteren Verlauf der Differenzierung kontinuierlich Makrophagen generiert und in den Überstand freigibt. Die Zellen können dann beispielsweise in einem wöchentlichen Turnus über einem Zeitraum von mehr als sechs Monaten geerntet werden (Abb. 1, [7]). Ausgehend von dieser Technologie und hin zu einer industriellen Herstellung der Zellen für die klinische Translation, haben wir gemeinsam mit den Leibniz Forschungslaboratorien für Biotechnologie und künstliche Organe (LEBAO, Dr. Zweigerdt) diesen Prozess in Bioreaktoren überführt. In proof-of-conceptStudien haben wir eine 250-ml-Plattform verwendet und damit im etablierten Prozess Makrophagen produziert. Derzeit sind wir in der Lage, in einem Prozessvolumen von ca.

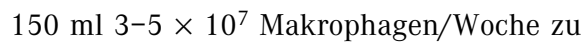


A
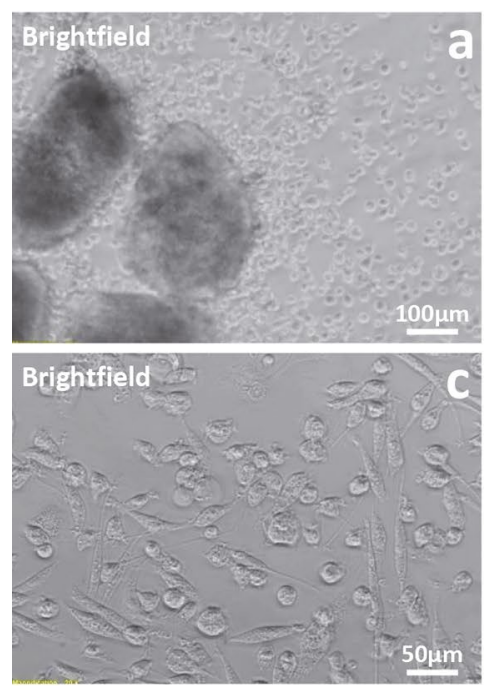
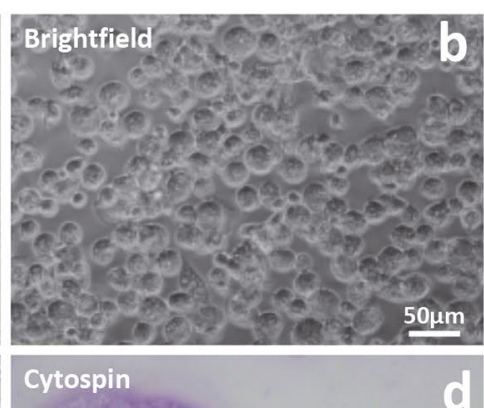

d
B

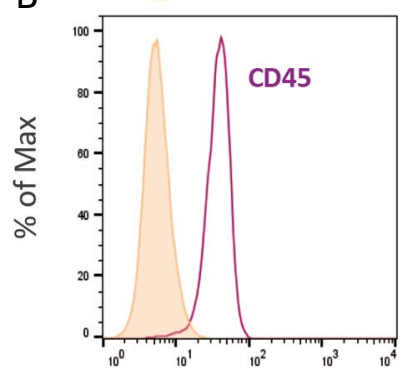

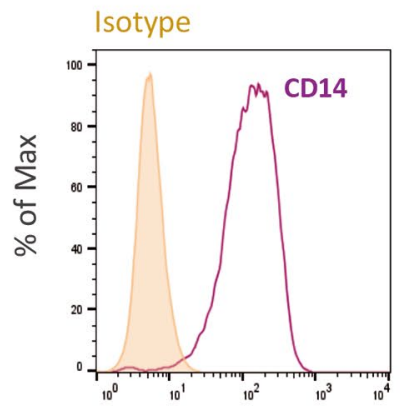
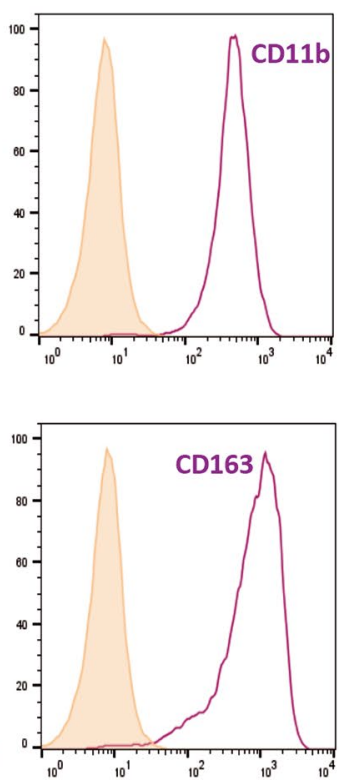

$\triangle$ Abb. 2: Generierung von Makrophagen aus humanen iPSZ. A, Humane Makrophagen werden kontinuierlich aus Zellaggregaten freigegeben (a) und haben in der Zellkultur eine typische Morphologie in Suspension nach der Ernte (b), nach terminaler Differenzierung mit M-CSF für weitere sieben Tage (c) sowie in Cytospin-Färbungen (d). B, Darüber hinaus exprimieren iPSZ-Makrophagen typische Oberflächenmarker wie CD45, CD11b, CD14 und CD 163 (violett).

produzieren [8]. Aufgrund der kontrollierbaren Bedingungen sowie der Möglichkeit, kritische Prozessparameter zu überwachen und direkt zu steuern, streben wir derzeit an, die Quantität der Zellen mit dem gleichen Prozessvolumen deutlich zu erhöhen. Im darauffolgenden Schritt steht dann das nächste Prozessvolumen von $500 \mathrm{ml}$ an, welches im weiteren Verlauf bis auf ein Volumen von 50 Liter erhöht werden kann. Die bisher im kleinen Prozessvolumen erzeugten Makrophagen weisen einen hohen Grad an Reinheit auf und haben die gleichen Funktionen wie Monozyten oder Makrophagen, die aus peripherem Blut gewonnen werden können. Wie oben erwähnt, werden die Makrophagen aus Zellaggregaten kontinuierlich freigegeben und besitzen eine Makrophagen-typische Morphologie (Abb. 2A). Darüber hinaus exprimieren die Zellen Makrophagen-typische Oberflächenmoleküle wie CD45, CD11b, CD14 sowie CD163 (Abb. 2B). Wir konnten sogar aufzeigen, dass die produzierten Zellen die Fähigkeit besitzen, stärker auf ihre Umwelt - wie z. B. Pathogene - zu reagieren als Makrophagen aus peripherem Blut. Diese Fähigkeit haben wir genutzt, um eine neuartige Makrophagen-basierte Immuntherapie zu etablieren und bakterielle Lungeninfektionen in einer Antibiotika-unabhängigen Therapie zu behandeln. In ersten Studien haben wir Gram-positive (Staphylococcus aureus) und Gram-negative (Pseudomonas aeruginsa, PsA) Bakterien genutzt, um eine akute Lungeninfektion in immunsupprimier- ten Mäusen zu erzeugen. Bei Studien mit PsA haben wir nach ersten Krankheitssymptomen die Makrophagen intra-pulmonal verabreicht. Im weiteren Verlauf konnten wir beobachten, dass sich der Krankheitszustand innerhalb weniger Stunden nach Gabe der Zellen signifikant verbessert und dass ca. 20 Stunden nach dem Zelltransfer fast keine Krankheitssymptome zu erkennen waren [8]. Derzeit untersuchen wir, wie wir diese Technologie bzw. diese hergestellten Makrophagen auch bei anderen schweren Infektionsverläufen in der Lunge einsetzen können.

\section{Literatur}

[1] Trapnell BC, Nakata K, Bonella F et al. (2019) Pulmonary alveolar proteinosis. Nat Rev Dis Primers 5: 16 [2] Jouanguy E, Dupuis S, Pallier A et al. (2000) In a novel form of IFN-gamma receptor 1 deficiency, cell surface receptors fail to bind IFN-gamma. J Clin Invest 105: 1429-1436 [3] Happle C, Lachmann N, Skuljec J et al. (2014) Pulmonary transplantation of macrophage progenitors as effective and long-lasting therapy for hereditary pulmonary alveolar proteinosis. Sci Transl Med 6: 250ra113

[4] Suzuki T, Arumugam P, Sakagami T et al. (2014) Pulmonary macrophage transplantation therapy. Nature 514 450-454

[5] Hahn K, Pollmann L, Nowak J et al. (2020) Human lentiviral gene therapy restores the cellular phenotype of autosomal recessive complete IFN-gammaR1 deficiency. Mol Ther Methods Clin Dev 17: 785-795

[6] Hetzel M, Mucci A, Blank P et al. (2018) Hematopoietic stem cell gene therapy for IFNgammaR1 deficiency protects mice from mycobacterial infections. Blood 131: 533-545 [7] Lachmann N, Ackermann M, Frenzel E et al. (2015) Large-scale hematopoietic differentiation of human induced pluripotent stem cells provides granulocytes or macrophages for cell replacement therapies. Stem Cell Reports 4: 282-296 [8] Ackermann M, Kempf H, Hetzel M et al. (2018) Bioreactor-based mass production of human iPSC-derived macrophages enables immunotherapies against bacterial airway infections. Nat Commun 9: 5088

Funding note: Open Access funding enabled and organized by Projekt DEAL. Open Access: Dieser Artikel wird unter der Creative Commons Namensnennu 4.0 International Lizenz veröffentlicht, welche die Nutzung, Vervielfältigung,
Bearbeitung, Verbreitung und Wiedergabe in jeglichem Medium und Format Bearbeitung, Verbreitung und Wiedergabe in jeglichem Medium und Fong
erlaubt, sofern Sie den/die ursprünglichen Autor(en) und die Quelle ordnungsgemäß nennen, einen Link zur Creative Commons Lizenz beifügen und ordnungsgemäß nennen, einen Link zur Creative Commons Lizenz beifu
angeben, ob Änderungen vorgenommen wurden. Die in diesem Artikel angeben, ob Anderungen vorgenommen wurden. Die in diesem Artikel
enthaltenen Bilder und sonstiges Drittmaterial unterliegen ebenfalls der genannten Creative Commons Lizenz, sofern sich aus der Abbildungslegende nichts anderes ergibt. Sofern das betreffende Material nicht unter der genannten Creative Commons Lizenz steht und die betreffende Handlung nich nach gesetzlichen Vorschriften erlaubt ist, ist für die oben aufgeführten Weiterverwendungen des Materials die Einwilligung des jeweiligen Rechteinhabers einzuholen. Weitere Details zur Lizenz entnehmen Sie bitte der

\section{Korrespondenzadresse:}

Prof. Dr. Nico Lachmann

Medizinische Hochschule Hannover

Klinik für Pädiatrische Pneumologie, Allergologie und Neonatologie OE 6710

Carl-Neuberg-Straße 1

D-30625 Hannover

Lachmann.nico@mh-hannover.de 